\title{
Dihydroartiminisin inhibits the growth and metastasis of epithelial ovarian cancer
}

\author{
BUCHU WU ${ }^{1,2}, \mathrm{KE} \mathrm{HU}^{1}, \mathrm{SHU}^{1}{ }^{1}, \mathrm{JING} \mathrm{ZHU}^{1}, \mathrm{LIYING} \mathrm{GU}^{1}$, \\ HAORAN SHEN ${ }^{1}$, BRETT D. HAMBLY ${ }^{2}$, SHISAN BAO ${ }^{1,2}$ and WEN DI ${ }^{1}$ \\ ${ }^{1}$ Department of Obstetrics and Gynecology, Ren-Ji Hospital, Shanghai Jiaotong University School of Medicine, Shanghai \\ 200127, P.R. China; ${ }^{2}$ Discipline of Pathology, School of Medical Science and Bosch Institute, University of Sydney, Australia
}

Received July 4, 2011; Accepted August 5, 2011

DOI: $10.3892 /$ or.2011.1505

\begin{abstract}
Dihydroartiminisin (DHA), the active component of a Chinese herb (Artemisia annua), has been utilised as an anti-malarial drug since ancient China. DHA has also been shown to inhibit proliferation of cancer in vitro. However, the capacity of DHA to inhibit the development of ovarian cancer is still unclear. The adhesion, invasion, and migration of human ovarian cancer cell line (HO8910PM) was determined following DHA treatment in vitro, using Matrigel coated plate, transwell membrane chamber, and wound healing models, respectively. A mouse ovarian cancer model was established by orthotopic inoculation of HO8910PM cell line in nude mice. The growth and metastasis in vivo was determined 8 weeks post-implantation in response to DHA treatment. The expression of phosphorylated focal adhesion kinase (pFAK) and matrix metalloproteinases (MMP-2 and MMP-9) was evaluated using Western blotting. The expression of Von Willebrand factor (vWF) and infiltration of macrophages were determined, using immunohistochemistry. DHA inhibits ovarian cancer cell proliferation, adhesion, migration and invasion in vitro in a dose-dependent manner, consistent with decreased expression of pFAK and MMP-2, but not MMP-9. DHA inhibited metastasis significantly in vivo, associated with reduced $\mathrm{vWF}$ expression and macrophage infiltration. In conclusion, DHA inhibits the development of ovarian cancer, in part via down-regulating pFAK, MMP-2, vWF and macrophage infiltration.
\end{abstract}

Correspondence to: Dr Wen Di, Department of Obstetrics and Gynaecology, Ren-Ji Hospital, Shanghai Jiaotong University School of Medicine, 1630 Dong Fang Road, Shanghai 200127, P.R. China E-mail: diwen163@163.com

Professor Shisan Bao, Discipline of Pathology, School of Medical Science and Bosch Institute, University of Sydney, NSW 2006 Australia

E-mail: bob.bao@sydney.edu.au

Key words: dihydroartiminisin, ovarian cancer, metastasis

\section{Introduction}

Artemisinin (Chinese name of Qing Hao $\mathrm{Su}$ ), the active component of a Chinese herb Artemisia annua, has been utilised as an anti-malarial drug since the Han Dynasty (AC800) in China $(1,2)$. Dihydroartemisinin (DHA), artesunate and artemether are analogues of artemisinin that are still widely used for anti-malarial therapy (1). Artemisinin and its derivatives have recently been shown to inhibit proliferation of malignant tumours in vitro (3-6), including breast (6) and ovarian cancer cells (7). Importantly, no cytotoxicity of DHA was detected in non-malignant cells $(6,7)$. Thus, DHA may be a useful anti-cancer drug, since it combines anti-cancer activity with minimal side effects, based on clinical data from anti-malaria therapy (3).

Ovarian cancer is a life-threatened disease in women, due to difficulty in early diagnosis and lack of effective treatment (8). Most advanced ovarian cancer patients develop peritoneal metastases with ascites, with $<30 \%$ 5-year survival rate (9). The mortality of ovarian cancer is the highest among all gynaecological cancers around the world (8). Thus, the need for new, more effective therapies is clear, particularly targeting growth and metastasis of ovarian cancer.

No studies have yet been undertaken on the capacity of DHA to inhibit ovarian cancer cell growth and metastasis in vivo. The aim of this study was to investigate the ability of DHA to inhibit the growth and metastasis of a human ovarian cancer cell line, with high metastatic potential, orthotopically implanted onto the ovaries of nude mice. Additionally, the possible underlying mechanisms of metastasis, proliferation and invasion have been determined. The data obtained suggest that DHA or its analogues may be an effective therapy for ovarian cancer, and provide important background for future both basic research and clinical therapeutic strategic development.

\section{Materials and methods}

Cell culture. The human ovarian epithelial cancer cell line HO8910PM with high metastasis potential, obtained from The Cell Bank, Chinese Academy of Sciences (10) was cultured in RPMI-1640 medium (Gibco, Shanghai, China), supplemented with 10\% FBC (Gibco) and penicillin/streptomycin (1:100, 
Sigma) in a humid atmosphere incubator with $5 \% \mathrm{CO}_{2}$ at $37^{\circ} \mathrm{C}$. Unless otherwise indicated, cells were grown to $70 \%$ confluence, then serum-starved overnight in serum-free RPMI-1640 medium prior to treatment (11).

Cell proliferation assay. HO-8910PM cell proliferation following DHA (Sigma) treatment for 24, 48 or $72 \mathrm{~h}$, was assessed by Cell Counting Kit-8 (CCK-8) (Dojindo, Shanghai, China), according to the manufacturer's instructions. Briefly, $3 \times 10^{3}$ cells were seeded into 96-well plates and incubated with DHA in the culture medium at various concentrations (250, $100,50,25,12.5,6.3,3.1,1.6$ and $0 \mu \mathrm{M})$, in triplicate. The control group was treated with $0.1 \%$ DMSO only, because DHA was dissolved in $0.1 \%$ DMSO. At each time point, $10 \mu 1$ CCK-8 was added to each well and cultured at $37^{\circ} \mathrm{C}$ for about $2 \mathrm{~h}$. The supernatant from the each well was collected for measurement of absorbance at $450 \mathrm{~nm}$, using MK3 microplate reader (Thermo). The inhibition rate of cell proliferation (see equation below) and $\mathrm{IC}_{50}(50 \%$ inhibiting concentration by non-linear regression fitting) was calculated. Proliferation rate $(\%)=[(\mathrm{Ac} / \mathrm{Ae}) /(\mathrm{Ac} / \mathrm{Ab})] \times 100 \%$. Ae is the absorbance of culture medium in experiment wells (cells treated with DHA), Ac the absorbance of culture medium in control wells (cells without treatment with DHA) and Ab the absorbance of culture medium in blank wells (culture medium only, without cells).

Adhesion assay. The 96-well plates, dry-coated with Matrigel (BD Biosciences, Shanghai, China), were blocked with $1 \%$ BSA for non-specific binding for $1 \mathrm{~h}$ at room temperature. HO-8910PM cells $\left(2 \times 10^{4}\right)$, pre-treated with DHA $(0,25$ or $50 \mu \mathrm{M})$ for $48 \mathrm{~h}$, were allowed to adhere to the Matrigel-coated well for $1 \mathrm{~h}$ at $37^{\circ} \mathrm{C}$. Non-adherent cells were removed by $5 \mathrm{X}$ washing with cold PBS. The adhesion rate was calculated by comparing the adherent cell numbers at different dosages vs. mock treatment.

Invasion assay. Cell motility was examined using a Boyden chamber apparatus. Briefly, HO-8910PM cells were harvested by trypsinization and resuspended in serum-free medium $\left(3 \times 10^{5} / \mathrm{ml}\right)$. Cells $\left(1 \times 10^{5} / \mathrm{ml}\right)$ were added into the upper wells of the Boyden chamber (Millicell, $8 \mu \mathrm{m}$ pore size, $12-\mathrm{mm}$ diameter, Millipore), while DHA $(0,25$, or $50 \mu \mathrm{M})$ was added to an inner cup of the Boyden chamber that had been coated with $50 \mu 1$ of Matrigel (1:6 dilution in serum-free medium). The RPMI-1640 medium with 20\% FBC was added to the lower chamber. Cell invasion into the Matrigel was determined, following 48 -h culture at $37^{\circ} \mathrm{C}$ in a humid atmosphere incubator containing $5 \% \mathrm{CO}_{2}$. The membrane containing invading cells was methanol fixed, stained with hematoxylin-eosin (HE), and quantified by light microscopy, after removing noninvading cells on the upper side of the membrane in the upper chamber with cotton swabs.

Migration assay. HO-8910PM cell migration was determined using a wound-healing motility assay. HO8910PM cells $\left(4 \times 10^{4}\right)$ were added to 6 -well plate with DHA $(0$ or $25 \mu \mathrm{M})$ and incubated for $48 \mathrm{~h}$. After scratching with a plastic cell scraper of fixed width, the monolayer of cells was washed 3 times with PBS to remove the detached cells. The remaining adherent cells were incubated in a humidified atmosphere with $5 \% \mathrm{CO}_{2}$ at $37^{\circ} \mathrm{C}$. Cell migration was determined by monitoring reduction in the width of the scaped zone at various time points for up to $48 \mathrm{~h}$; the migration rate was calculated from the migration distance at different dosages compared to mock treatment, in triplicate.

Western blot analysis. HO-8910PM cells with treatment of DHA $(0,12.5,25,50 \mu \mathrm{M})$ for $48 \mathrm{~h}$ were harvested by washing with cold PBS. The HO-8910PM cells were lysed by RIPA (Roche, Shanghai, China) on ice for $30 \mathrm{~min}$. The supernatant was collected after centrifugation at 14,000 rpm for $10 \mathrm{~min}$ at $4^{\circ} \mathrm{C}$. The protein concentration was determined by the Bradford method (12). Briefly, Western blot analysis was performed by transferring the protein samples to NC membrane (Millipore), after separation by electrophoresis on an $8 \%$ SDS-polyacrylamide gel. Non-specific binding was blocked with $10 \%$ dry milk in TBST for $1 \mathrm{~h}$ at room temperature. The membranes were then incubated with primary antibodies against human MMP-2 (1:1000), MMP-9 (1:500), p-FAK (1:500), $\beta$-actin (1:1000; housekeeping protein) (Cell Signalling, Shanghai, China) and FAK (1:250) (R\&D, Shanghai, China), in TBST with $5 \%$ milk powder overnight at $4^{\circ} \mathrm{C}$. The secondary antibodies were applied at room temperature for $1 \mathrm{~h}$, after 3 times washing with TBST. The expression of specific proteins was detected by an ECL Kit (Millipore) following the instructions from the manufacturer and visualized by autoradiography with Hyper film (Millipore).

Determination of metastasis. Female athymic BALB/c nude mice, 5-week old, were obtained from the Chinese Academy of Sciences, as previously described (13). All animals were housed under conventional laboratory conditions with food and water ad libitum. Experiments adhered to the guidelines of the Shanghai Jiaotong University School of Medicine, Animal Ethics Committee (AEC).

Establishment of ovarian cancer in vivo. Nude mice were inoculated with $2 \times 10^{6} \mathrm{HO}-8910 \mathrm{PM}$ cells in $100 \mu \mathrm{l}$ of PBS on their back, to establish a viable solid tumour. The established tumour from the donor was then dissected into $1 \mathrm{~mm}^{3}$ pieces and, during laparotomy under anesthesia, one piece was implanted orthotopically on the right ovarian capsule of the recipient nude mouse, using a 6-0 surgical suture. The abdominal wall was stitched with a 3-0 silk suture after returning the ovary to its original location.

To evaluate the anti-metastatic effect of DHA on ovarian cancer, a total of 10 female nude mice (5-week old) were randomly divided into experimental or control groups. Two weeks post-tumour inoculation, DHA (500 mM), dissolved in $400 \mu 1$ DMSO in normal saline (1:30), was given intraperitoneally at $50 \mathrm{mg} / \mathrm{kg}$ dose, three times a week for 4 weeks; while the mock treated group was given vehicle only.

The size and number of the local and metastatic tumours were determined by mean tumour volume [MTV calculated by the equation: $\mathrm{MTV}=\mathrm{XY}^{2} / 2$, where $\mathrm{X}$ is the longitudinal and $\mathrm{Y}$ is the horizontal measurements $(\mathrm{mm})$ of orthogonal measurements] (14) on 8 weeks post-implantation with/without treatments. For histopathological examination, the tissues were fixed in $10 \%$ neutral-buffered formalin and wax embedded. The sections were cut at $5 \mu \mathrm{m}$ and stained with H\&E. 


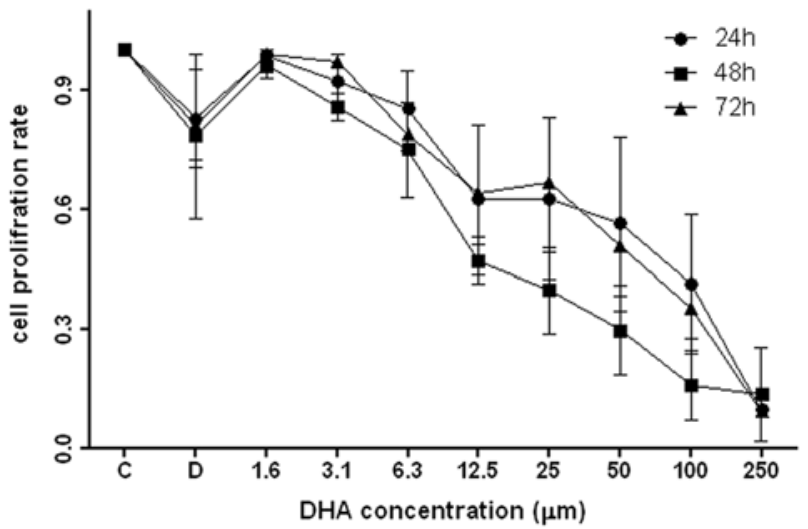

Figure 1. Proliferation rate of HO-8910PM cells following DHA treatment for 24, 48 and 72 h. Mean \pm SEM. ${ }^{*} \mathrm{P}<0.05,{ }^{* *} \mathrm{P}<0.01$ and ${ }^{* * *} \mathrm{P}<0.001$. Cytotoxicity was determined by CCK- 8 as described.

Immunohistochemistry. Paraffin sections $(5 \mu \mathrm{m})$ were incubated with rat anti-F4/80 (WEHI, Melbourne, Australia) or vWF (Dako, Australia) antibodies followed by a horseradish peroxidase (HRP)-conjugated rabbit anti-rat antibody (Dako), according to the manufacturer's instructions, as previously described (15). All sections were then visualized with 3,3' diaminobenzidine (DAB) (Dako), and lightly counterstained with hematoxylin.

Immunohistochemical quantifications were performed on digitized images using image analysis software ImagePro Plus 4.5 (Diagnostic Instruments, USA). The presence of monocytes/macrophages was assessed using anti-F4/80 antibody and the total number of positive cells were determined by [100\% x (number of positive nuclei/total number of nuclei)]. vWF positive cells were calculated and compared by density with ImagePro Plus 4.5.

Statistical analysis. Each experiment was repeated twice. All data are expressed as means \pm standard error or division of the mean (SEM or SD). Data analysis was carried out by Student's t-tests and two-way ANOVA using the statistical program in GraphPad Prism Version 4.0 (GraphPad Software, San Diego, CA, USA). A value of $\mathrm{P}<0.05$ was considered statistically significant.

\section{Results}

DHA inhibits the growth of human HO-8910PM cells in vitro. DHA significantly inhibited the cell proliferation of HO-8910PM cells in a dose-dependent manner (range from $0-250 \mu \mathrm{M})$ at 24,48 and $72 \mathrm{~h}$ in vitro (Fig. 1). Significantly greater inhibition of proliferation of HO-8910PM cells was observed following 48-h treatment with DHA in the range 12.5-100 $\mu \mathrm{M}$, compared to 24 and $72-\mathrm{h}$ treatment. DHA $(25-50 \mu \mathrm{M})$ exhibited approximately $50 \%$ growth inhibition of HO-8910PM cells $(\mathrm{P}<0.01)$.

DHA decreases adhesion. Matrigel contains almost all of the extra-cellular matrix (ECM) components and is a water soluble basement membrane preparation extracted from Engelbreth-Holm-Swarm (EHS) mouse sarcoma (16). DHA

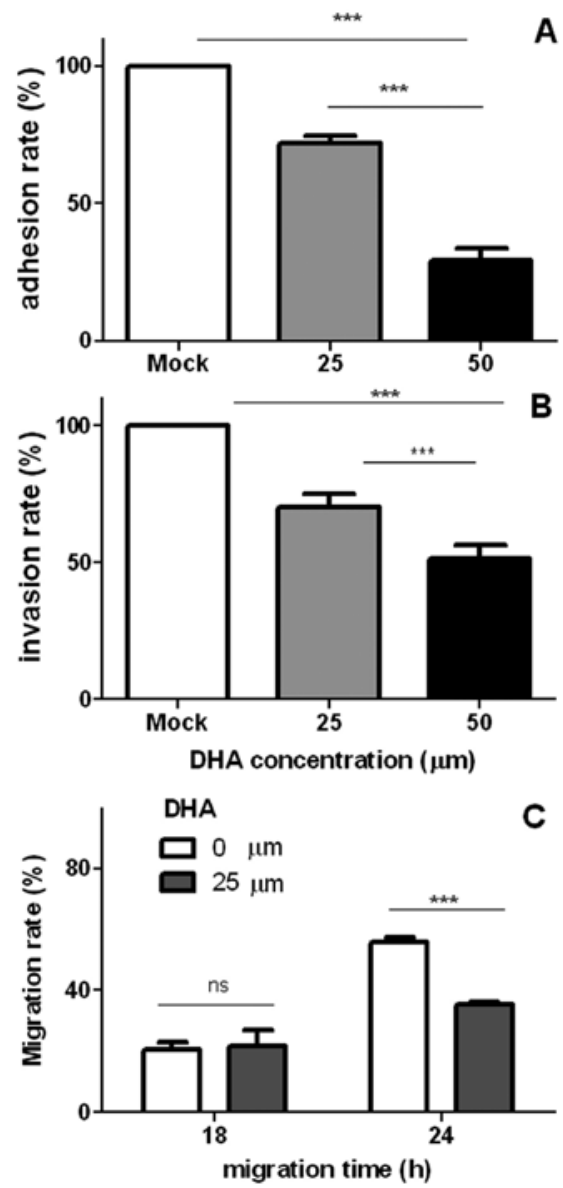

Figure 2. Cellular adhesion (A), invasion (B) and migration (C) of HO-8910PM cells were inhibited by DHA at different dosages. The adhesion rate was determined by using CCK-8. Cellular invasion rate was determined using the Transwell apparatus method. Cellular migration rate was monitored by phase-contrast microscopy (x100) following 18 and 24-h DHA treatment. (D) DMSO mean \pm SEM. ${ }^{*} \mathrm{P}<0.05,{ }^{* *} \mathrm{P}<0.01$ and ${ }^{* * *} \mathrm{P}<0.001$.

significantly inhibited cell adhesion of HO-8910PM cells in a dose-dependent manner $(\mathrm{P}<0.001)$, by approximately $25 \%$ at $25 \mu \mathrm{M}$ and $40 \%$ at $50 \mu \mathrm{M}$, compared to the mock-treated groups (Fig. 2A).

DHA inhibits invasion. Invasion is among the most significant biological characteristics of malignant tumours. The capacity of DHA to inhibit tumour invasion was determined in HO-8910PM cells using a cell invasion assay. DHA treatment $(0-50 \mu \mathrm{M})$ resulted in a significant $(\mathrm{P}<0.001)$ dose-dependent decline in adhesion rate, demonstrating decreased invasive ability of the HO-8910PM cells (Fig. 2B).

DHA inhibits migration. After $48 \mathrm{~h}$ of wound healing, our data showed that DHA significantly inhibited the migration of HO-8910PM cells in a dose-dependent manner (Fig. 2C). Mock-treated HO-8910PM cells had filled 30\% of the gap, while cells treated with DHA $25 \mu \mathrm{M}$ filled only $10 \%$ (Fig. 2C).

DHA regulates FAK, $p-F A K, M M P-2$ and $M M P-9$. The active, phosphorylated form of focal adhesion kinase (p-FAK) is a non-receptor protein tyrosine kinase that is involved in cell-ECM interactions, migration, proliferation, survival 


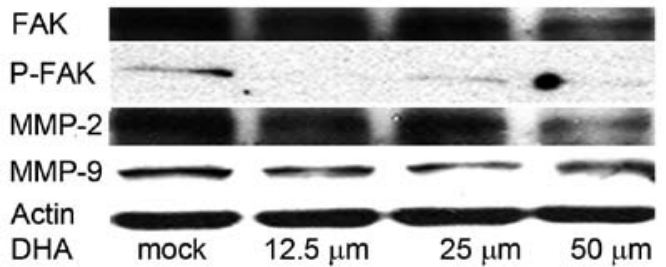

Figure 3. The levels of protein expression in HO8910-PM ovarian cancer cells following Mock and DHA treatments: FAK, pFAK, MMP-2, MMP-9; $\beta$-actin was used as control.
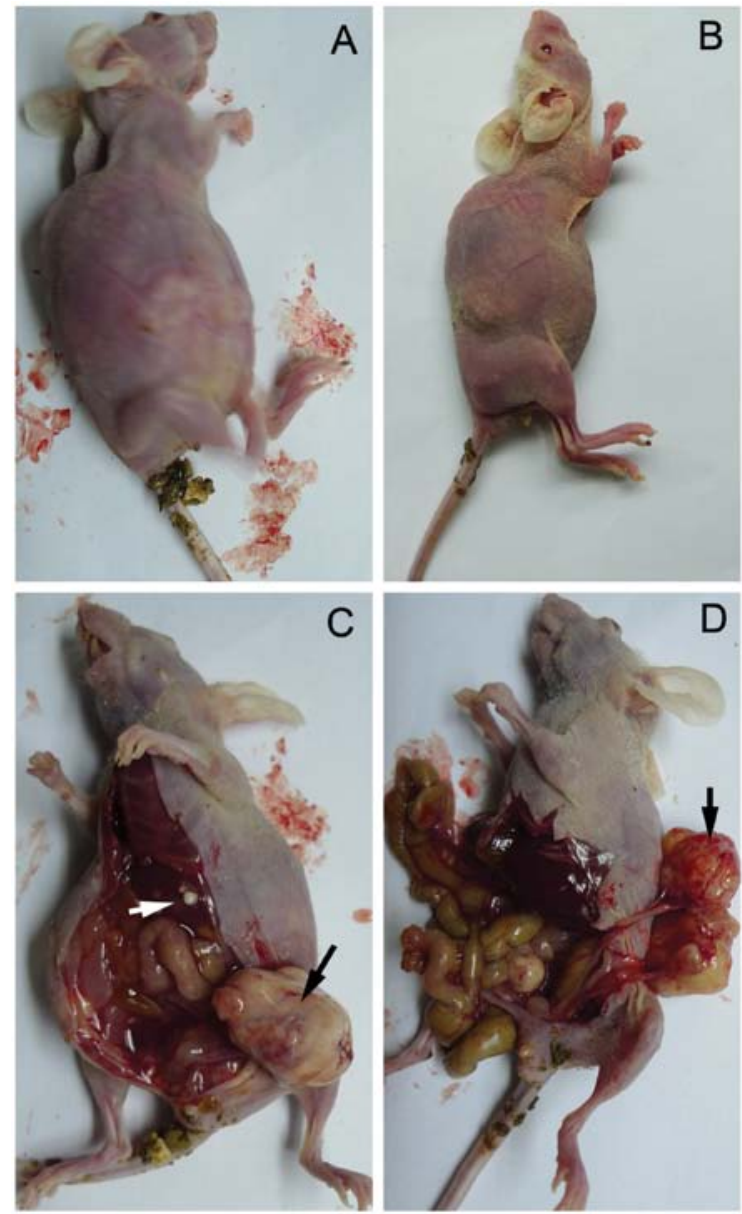

Figure 4. The metastasis of HO-8910PM ovarian cancer cells inoculated in the nude mice following mock- (A and C) or DHA-treatment (B and D). Inoculated ovarian tumour (black arrow) metastasised to intestine, liver, and peritoneum (white arrows). Fewer and smaller metastases were observed in the DHA-treated group (C vs. D).

and metastasis $(17,18)$. The extent of p-FAK overexpression correlates with increased metastasis and decreased survival in human ovarian cancer $(17,18)$. Using Western blot assay, DHA (at various dosages $12.5,25,50 \mu \mathrm{M}, 48$-h treatment) downregulated p-FAK expression significantly in a dose-dependent manner, while FAK expression changed only at $50 \mu \mathrm{M}$ DHA treatment (Fig. 3).

MMP-2 and MMP-9 degrades components of the extracellular matrix (ECM) proteins, contributing to tumour invasion and metastasis $(19,20)$. DHA $(12.5,25,50 \mu \mathrm{M}, 48$-h treatment) decreased MMP-2 expressions at the protein level in

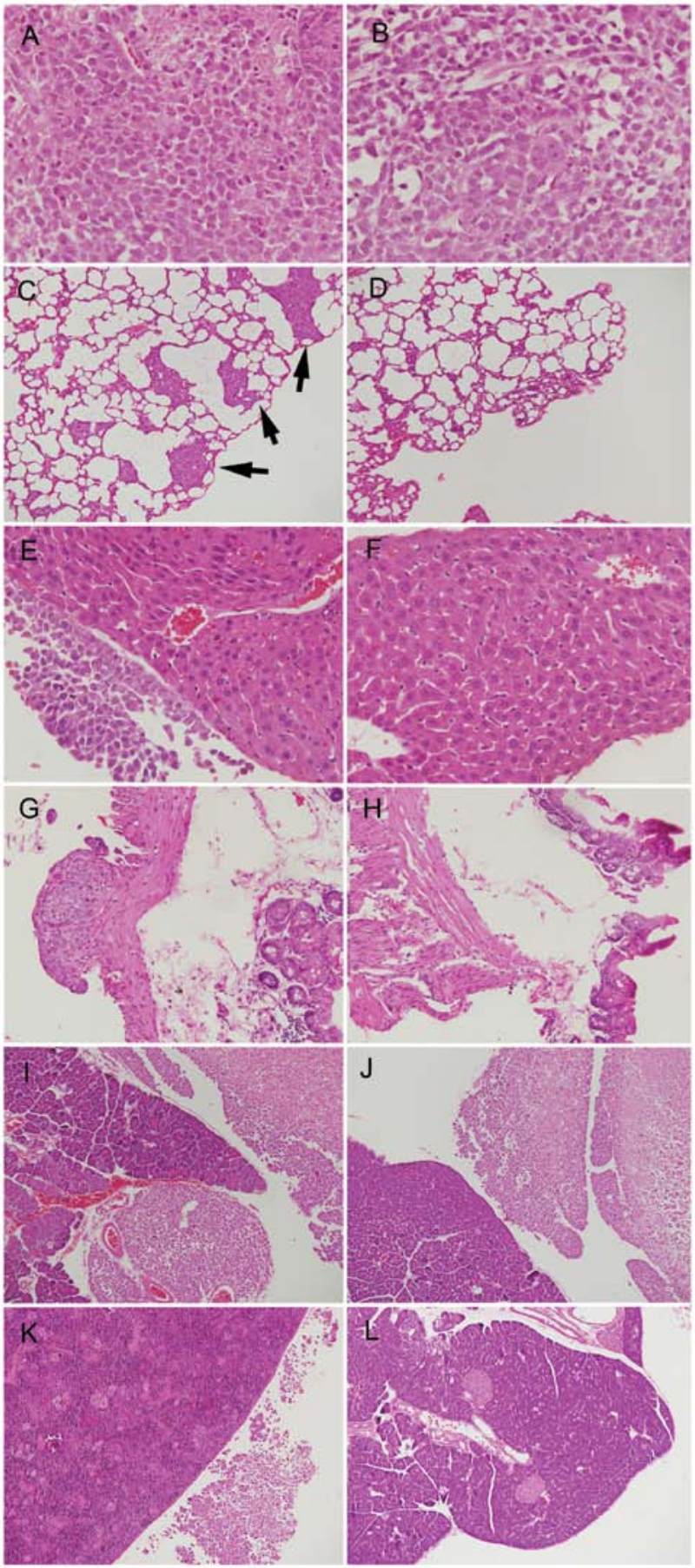

Figure 5. Histopathology of DHA and mock-treated groups from HO-8910PM inoculated ovarian tumour. The slides were $\mathrm{HE}$ stained and visualized at $\mathrm{x} 4$, $\mathrm{x} 10$ or $\mathrm{x} 20$. Histology of the inoculated ovarian tumour showed malignant papillary serous adenocarcinoma (A and B). Tumour cells metastasize to the lung $(\mathrm{C}$ and $\mathrm{D})$ with arrows, liver $(\mathrm{E}$ and $\mathrm{F})$, intestine $(\mathrm{G}$ and $\mathrm{H})$, spleen (I and $\mathrm{J})$ and pancreas ( $\mathrm{K}$ and $\mathrm{L})$.

HO-8910PM cells in a dose-dependent manner, but did not alter MMP-9 expressions, using a Western blot assay (Fig. 3).

DHA inhibits metastasis in vivo. All the mice $(100 \%, 10 / 10)$ developed bloody ascites two weeks post orthotopic tumour implantation in both DHA $(50 \mathrm{mg} / \mathrm{kg})$ and mock treated groups (Fig. 4A and B). No significant difference in orthotopic ovary tumour size was observed between DHA and mock treated groups $\left(1078.2 \pm 549.6 \mathrm{~mm}^{3}\right.$ vs. $\left.1154.9 \pm 786.5 \mathrm{~mm}^{3}\right)$ 


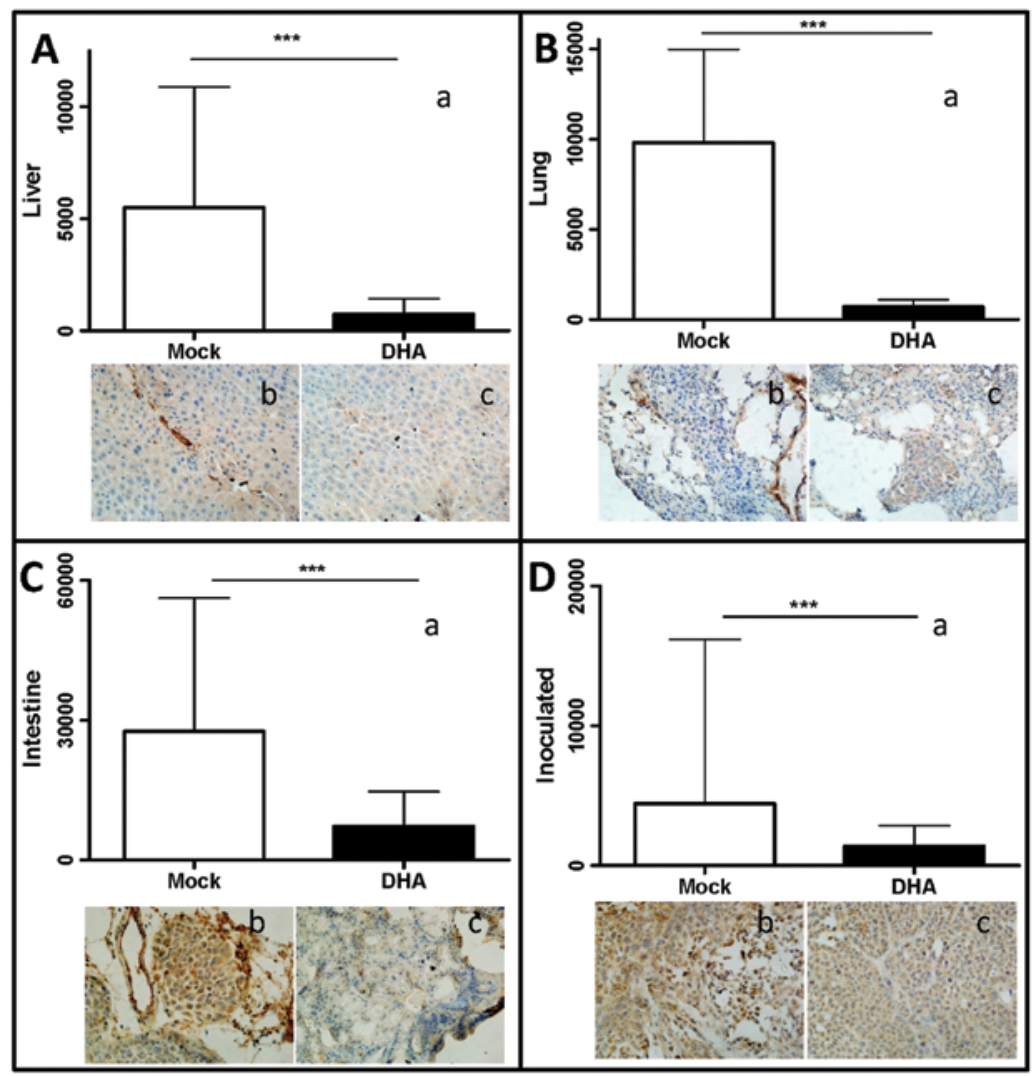

Figure 6. vWF immunohistostaining of inoculated and metastatic tumour following Mock (b) and DHA (c) treatment. The expression of vWF (a) was significantly decreased in metastases to the liver (A), lung (B) and intestine (C), and in the inoculated site (D) following treatment with DHA compared to Mock treatment $(\mathrm{P}<0.001)$.

(black arrows, Fig. 4C and D). Metastases were detected in all mice (5/5) of mock treated group; in the peritoneum (5/5), contralateral ovary (2/5), intestine (5/5), liver (2/5), and spleen (1/5) (white arrows, Fig. 4C). Metastases in DHA treated group was: peritoneum (3/5), intestine (5/5) and liver metastasis (3/5) (white arrow, Fig. 4D).

Histopathology. The orthotopic tumours demonstrated similar histopathological features to malignant papillary serous adenocarcinoma of the ovary (Fig. 5A and B). No obvious metastatic lesion was observed macroscopically in the lung with/without DHA treatment. However, substantially fewer metastases were detected under microscopic examination in the lung from the mock treated compared to the DHA treated animals (Fig. 5C and D). There were significantly more and larger metastases observed in the liver from the mock treated mice compared to the DHA treated animals (Fig. 5E and F). There were macroscopic metastatic lesions detected in the intestine from both mock and DHA treated animals, but more invasive lesions were detected in the mock treated group, that were larger in size and more deeply invasive, compared to the DHA treated group (Fig. 5G and H). Metastases were observed on the peritoneal covering of the spleen, but no direct invasion of the pancreas was detected (Fig. 5I and J) for either mock or the DHA treated group. Similar numbers of metastatic lesions were observed on the capsular surface of the pancrease, but microscopic analysis revealed that in the mock treated animals metastases were more invasive, through the splenic capsule, and larger (Fig. 5K), compared to the DHA treated spleens (Fig. 5L).

Immunohistochemistry staining for $v W F$ and macrophage. To investigate whether DHA inhibits neovascularisation, vWF was evaluated using immunohistochemistry. DHA suppressed vWF expression significantly in cancer lesions (liver, lung, intestine and the inoculated site) compared with the mock treatment group (Fig. 6). The vWF expression in the liver, lung, intestine and the inoculated tumour following DHA treatment was $\sim 20, \sim 10, \sim 30$ and $\sim 50 \%$ of the values obtained for the mock treated group $(\mathrm{P}<0.001)$, respectively.

Furthermore, to investigate the relationship between macrophage infiltration and tumour metastasis, the number of infiltrating macrophages was determined. There was a significant suppression of macrophage infiltration in the metastatic lesions following DHA treatment, compared to that of the mock treated group. The suppressed recruitment of macrophages in the liver, lung, intestine and inoculated tumour from DHA treated group was $\sim 20, \sim 10, \sim 30$ and $\sim 50 \%$, compared to that of the mock treated group $(\mathrm{P}<0.001)$, respectively (Fig. 7).

\section{Discussion}

The current study demonstrated that DHA inhibits the growth of human epithelial ovarian cancer in vitro by reducing proliferation, adhesion, invasion and migration via decreasing expression of MMP-2 and p-FAK in a dose- and time-depen- 


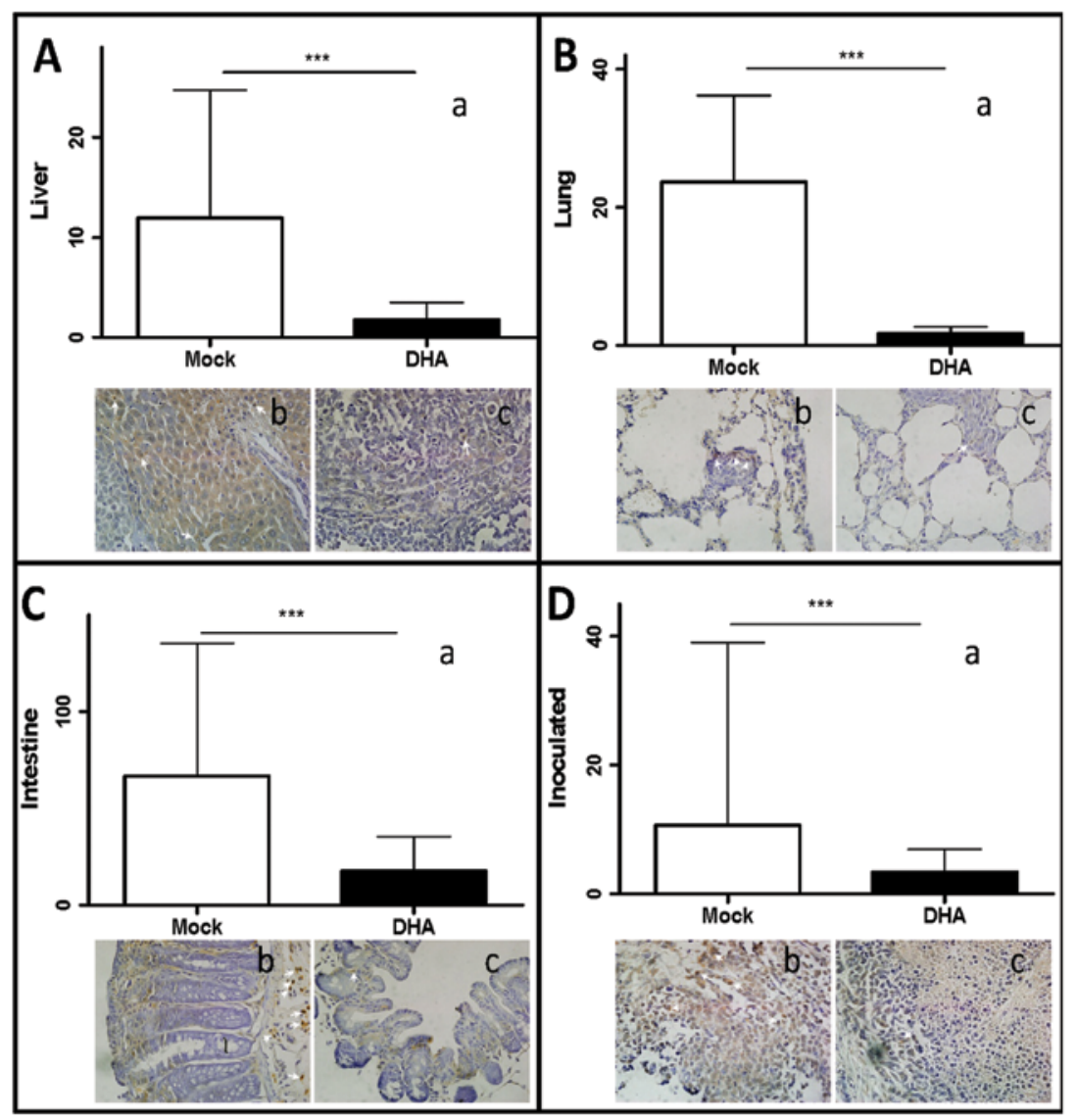

Figure 7. Macrophage immunohistostaining (white arrows) of inoculated and metastatic tumour following Mock (b) or DHA (c) treatment. Macrophage infiltration (a) was significantly decreased in metastases to the liver (A), lung (B) and intestine (C), and in the inoculated site (D) following treatment with DHA compared to Mock treatment $(\mathrm{P}<0.001)$.

dent manner. Furthermore, DHA suppressed the growth and metastasis of orthotopic tumour in vivo, correlating with the down-regulation of macrophage infiltration and vWF expression.

The overall development of cancer and progression to metastasis is closely dependent on adhesion, migration, invasion and angiogenesis $(13,21)$. Cell adhesion is critical for cancer cell proliferation, migration, invasion, as well as metastasis (21). After detachment, tumour cells separate and adhere through membrane receptors for invasion and migration $(13,21)$. Our data show that DHA inhibits ovarian cancer via reducing the proliferation and adhesion of ovarian epithelial cancer cells in vitro. These data are consistent with other findings that DHA is able to inhibit the proliferation of several other malignant cell lines (3-7,22-25).

Invasion and migration of cancer cells are essential steps during tumourgenesis following adhesion. Our results showed that DHA inhibited cell migration and invasion significantly in human ovarian cancer cells in a dose-dependent manner, which is in line with the findings that DHA inhibited migration of melanoma cells (22) and sarcoma cells (26). Tan et al, however, reported that DHA had no effect on human ovarian cancer cell line SKOV3 (27). The discrepancy between our findings and their results may be due to different characteristics of these cancer cell lines, and/or different dosages. Our ovarian cancer cell line (HO8910PM) has higher metastatic potential compared to other ovarian cancer cell lines (10), and is consequently more suitable for investigating the effect of DHA on metastasis.

DHA was also able to inhibit ovarian cancer cell (HO8910PM) growth and metastasis, following ovarian orthotopic transplantation, in vivo, probably via inhibiting proliferation, which is supported by our findings in vitro, and also consistent with other reports in papilloma virusexpressing epithelial (28), lung cancer (29), pancreas cancer (30) and hepatoma (23) in vivo. The dosage of DHA from our current experiment is $50 \mathrm{mg} / \mathrm{kg}$, which is about the mid-range of dosages used in the literature. Future studies with larger numbers of mice will determine the optimal therapeutic dose of DHA.

Molecules and pathways shown to be involved with the processes of adhesion, migration and invasion of tumour cells include laminin, MMPs/TIMPs, integrins and FAK $(19,31)$. FAK is important in cell-ECM interactions and its phosphorylated form (pFAK) plays an important role in malignant cell migration, proliferation, and survival $(31,32)$. Our observation that pFAK, but not FAK, was reduced following DHA treatment is consistent with inhibition of metastasis of our ovarian cancer cell line in vivo. Thus, our findings suggest that DHA inhibits ovarian cancer metastasis via the pFAK pathway. Up-regulated expression of MMPs is required for cancer cell invasion and metastasis through the extracellular matrix and basement membrane $(13,19,33)$. The levels of MMP-2 and MMP-9 expression by tumours are closely related to their 
metastatic potential (19). Our study demonstrated that DHA inhibit production of MMP-2 only, suggesting that DHA may differentially regulate MMPs and inhibit ovarian cancer metastasis via the MMP-2 pathway. Our data are consistent with the previous observation that inhibition of cell migration by artemisinin (an analogue of DHA) was associated with decreased expression of MMP-2 (22). On the other hand, MMP-9 is highly up-regulated in ovarian cancer, particularly in metastases (19). Our data showed that MMP-9 production was not inhibited by DHA treatment, while Anfosso et al found that artermisinin inhibited the expression of MMP-9 in cancer cells (33). The difference between these findings may be due to different cell lines and/or different treatments. Taken together, the MMP family may be a key target of DHA in the treatment of human ovarian cancer.

Angiogenesis is another objective factor predicting malignant prognosis (34) for overall and disease-free survival in advanced ovarian cancer $(35,36)$. It has been demonstrated in our study that DHA significantly suppressed expression of vWF, reflecting reduced neovascularisation, in both the inoculated site and metastatic tissues, compared to mock treatment. These findings provide evidence that the anti-tumour effects of DHA in ovarian cancer may also be mediated through inhibition of neovascularisation.

Finally, macrophages in the tumour microenvironment are designated as tumour-associated macrophages, and are associated with several pro-tumour functions, e.g. secretion of growth factors and matrix proteases, promotion of angiogenesis and suppression of adaptive immunity $(37,38)$. Macrophages also contribute to tumour dissemination via up-regulating tumour cell adhesion molecules on the peritoneal mesothelium, and releasing growth factors and invasive proteases $(39,40)$. These reports are consistent with our findings that DHA substantially suppresses macrophage infiltration in both the inoculated and metastatic sites (liver, lung and intestine), compared to the mock treatment group. Thus, suppression of macrophage infiltration may be another mechanism by which DHA inhibits metastasis in vivo, and suggests another mechanism of carcinogenesis that could be a therapeutic target.

In conclusion, DHA suppresses human epithelial ovarian cancer cell proliferation, adhesion, invasion and migration in vitro, via the FAK and MMP pathways; and also inhibits growth and metastasis of ovarian cancer in vivo, via inhibiting neovascularisation and macrophage infiltration. Based on the use of DHA as an anti-malarial, it has an excellent safety profile and has few side effects, and has minimal effects on the growth of normal ovarian cells in vitro $(6,7)$. Thus, a DHA-based drug may become a reliable alternative for the treatment of ovarian cancer, compared to the current standard anti-cancer therapy. Further details of the precise mechanism of action of this class of drugs remains to be explored.

\section{Acknowledgements}

This research is supported by grants from Hundred Talent program; Shanghai Jiaotong University School of Medicine; Overseas Exchange PhD Fellowship, Shanghai Jiaotong University; The Department of Science and Technology; Shanghai Municipal (08XD14027); the National Natural Science Foundation (30772306), and an Award of Outstanding
Young Academics Foundation of Shanghai Universities (JDY08042). The authors acknowledge assistance from the Histopathology Laboratory, Discipline of Pathology, The University of Sydney.

\section{References}

1. Meshnick SR: Artemisinin: mechanisms of action, resistance and toxicity. Int J Parasitol 32: 1655-1660, 2002.

2. O'Neill PM: Medicinal chemistry: a worthy adversary for malaria. Nature 430: 838-839, 2004.

3. Efferth T, Dunstan H, Sauerbrey A, Miyachi H and Chitambar CR: The anti-malarial artesunate is also active against cancer. Int $\mathrm{J}$ Oncol 18: 767-773, 2001.

4. Huang XJ, Ma ZQ, Zhang WP, Lu YB and Wei EQ: Dihydroartemisinin exerts cytotoxic effects and inhibits hypoxia inducible factor-1alpha activation in C6 glioma cells. J Pharm Pharmacol 59: 849-856, 2007.

5. Nam W, Tak J, Ryu JK, et al: Effects of artemisinin and its derivatives on growth inhibition and apoptosis of oral cancer cells. Head Neck 29: 335-340, 2007.

6. Singh NP and Lai H: Selective toxicity of dihydroartemisinin and holotransferrin toward human breast cancer cells. Life Sci 70: 49-56, 2001.

7. Chen T, Li M, Zhang R and Wang H: Dihydroartemisinin induces apoptosis and sensitizes human ovarian cancer cells to carboplatin therapy. J Cell Mol Med 13: 1358-1370, 2009.

8. Bertone-Johnson ER: Epidemiology of ovarian cancer: a status report. Lancet 365: 101-102, 2005.

9. Jemal A, Siegel R, Ward E, Hao Y, Xu J and Thun MJ: Cancer statistics, 2009. CA Cancer J Clin 59: 225-249, 2009.

10. Shenhua X, Lijuan Q, Hanzhou N, et al: Establishment of a highly metastatic human ovarian cancer cell line (HO-8910PM) and its characterization. J Exp Clin Cancer Res 18: 233-239, 1999.

11. Dai L, Gu L, Ding C, Qiu L and Di W: TWEAK promotes ovarian cancer cell metastasis via NF-kappaB pathway activation and VEGF expression. Cancer Lett 283: 159-167, 2009.

12. Bradford MM: A rapid and sensitive method for the quantitation of microgram quantities of protein utilizing the principle of protein-dye binding. Anal Biochem 72: 248-254, 1976.

13. Zhang J and Wang B: Arsenic trioxide $(\operatorname{As}(2) \mathrm{O}(3))$ inhibits peritoneal invasion of ovarian carcinoma cells in vitro and in vivo. Gynecol Oncol 103: 199-206, 2006.

14. Zhang S, Lin QD and Di W: Suppression of human ovarian carcinoma metastasis by the metastasis-suppressor gene, BRMS1. Int J Gynecol Cancer 16: 522-531, 2006.

15. Xu Y, Hunt NH and Bao S: The role of granulocyte macrophage-colony-stimulating factor in acute intestinal inflammation. Cell Res 18: 1220-1229, 2008.

16. Yang JZ, Ho AL, Ajonuma LC, et al: Differential effects of Matrigel and its components on functional activity of CFTR and $\mathrm{ENaC}$ in mouse endometrial epithelial cells. Cell Biol Int 27: 543-548, 2003.

17. Schlaepfer DD, Mitra SK and Ilic D: Control of motile and invasive cell phenotypes by focal adhesion kinase. Biochim Biophys Acta 1692: 77-102, 2004.

18. McLean GW, Carragher NO, Avizienyte E, Evans J, Brunton VG and Frame MC: The role of focal-adhesion kinase in cancer: a new therapeutic opportunity. Nat Rev Cancer 5: 505-515, 2005 .

19. Hoekstra R, Eskens FA and Verweij J: Matrix metalloproteinase inhibitors: current developments and future perspectives. Oncologist 6: 415-427, 2001.

20. Lengyel E, Schmalfeldt B, Konik E, et al: Expression of latent matrix metalloproteinase 9 (MMP-9) predicts survival in advanced ovarian cancer. Gynecol Oncol 82: 291-298, 2001.

21. Cattaruzza S and Perris R: Proteoglycan control of cell movement during wound healing and cancer spreading. Matrix Biol 24: 400-417, 2005.

22. Buommino E, Baroni A, Canozo N, et al: Artemisinin reduces human melanoma cell migration by down-regulating alpha $\mathrm{V}$ beta 3 integrin and reducing metalloproteinase 2 production. Invest New Drugs 27: 412-418, 2009.

23. Hou J, Wang D, Zhang R and Wang H: Experimental therapy of hepatoma with artemisinin and its derivatives: in vitro and in vivo activity, chemosensitization, and mechanisms of action. Clin Cancer Res 14: 5519-5530, 2008. 
24. Jiao Y, Ge CM, Meng QH, Cao JP, Tong J and Fan SJ: Dihydroartemisinin is an inhibitor of ovarian cancer cell growth. Acta Pharmacol Sin 28: 1045-1056, 2007.

25. Lai $\mathrm{H}$ and Singh NP: Oral artemisinin prevents and delays the development of 7,12-dimethylbenz[a]anthracene (DMBA)induced breast cancer in the rat. Cancer Lett 231: 43-48, 2006.

26. Hwang YP, Yun HJ, Kim HG, Han EH, Lee GW and Jeong HG: Suppression of PMA-induced tumor cell invasion by dihydroartemisinin via inhibition of PKCalpha/Raf/MAPKs and NF-kappaB/AP-1-dependent mechanisms. Biochem Pharmacol 79: 1714-1726, 2010.

27. Tan XJ, Lang JH, Plouet J, Wu M and Shen K: [Effects of dihydroartiminisin on the adhesion, migration, and invasion of epithelial ovarian cancer cells]. Zhonghua Yi Xue Za Zhi 88 2642-2646, 2008.

28. Disbrow GL, Baege AC, Kierpiec KA, et al: Dihydroartemisinin is cytotoxic to papillomavirus-expressing epithelial cells in vitro and in vivo. Cancer Res 65: 10854-10861, 2005.

29. Zhou HJ, Zhang JL, Li A, Wang Z and Lou XE: Dihydroartemisinin improves the efficiency of chemotherapeutics in lung carcinomas in vivo and inhibits murine Lewis lung carcinoma cell line growth in vitro. Cancer Chemother Pharmacol 66: 21-29, 2010.

30. Wang SJ, Gao Y, Chen $\mathrm{H}$, et al: Dihydroartemisinin inactivates NF-kappaB and potentiates the anti-tumor effect of gemcitabine on pancreatic cancer both in vitro and in vivo. Cancer Lett 293 99-108, 2010.

31. Parsons JT, Martin KH, Slack JK, Taylor JM and Weed SA: Focal adhesion kinase: a regulator of focal adhesion dynamics and cell movement. Oncogene 19: 5606-5613, 2000
32. Provenzano PP and Keely PJ: The role of focal adhesion kinase in tumor initiation and progression. Cell Adh Migr 3: 347-350, 2009.

33. Anfosso L, Efferth T, Albini A and Pfeffer U: Microarray expression profiles of angiogenesis-related genes predict tumor cell response to artemisinins. Pharmacogenomics J 6: 269-278, 2006.

34. Weidner N, Folkman J, Pozza F, et al: Tumor angiogenesis: a new significant and independent prognostic indicator in early-stage breast carcinoma. J Natl Cancer Inst 84: 1875-1887, 1992.

35. Brem S, Cotran R and Folkman J: Tumor angiogenesis: a quantitative method for histologic grading. J Natl Cancer Inst 48: 347-356, 1972.

36. Weidner N, Semple JP, Welch WR and Folkman J: Tumor angiogenesis and metastasis - correlation in invasive breast carcinoma. N Engl J Med 324: 1-8, 1991.

37. Coussens LM and Werb Z: Inflammation and cancer. Nature 420: 860-867, 2002

38. Lewis CE and Pollard JW: Distinct role of macrophages in different tumor microenvironments. Cancer Res 66: 605-612, 2006.

39. Jonjic N, Peri G, Bernasconi S, et al: Expression of adhesion molecules and chemotactic cytokines in cultured human mesothelial cells. J Exp Med 176: 1165-1174, 1992.

40. Wang E, Ngalame Y, Panelli MC, et al: Peritoneal and subperitoneal stroma may facilitate regional spread of ovarian cancer. Clin Cancer Res 11: 113-122, 2005. 